\title{
Advances Towards 4J Lattice-Matched including Dilute Nitride Subcell for Terrestrial and Space Applications.
}

\author{
M. Ochoa, I. García, I. Lombardero, L. Ayllón, L. Cifuentes, I. Rey-Stolle and C. Algora \\ A. D. Johnson and J. I. Davies \\ K.H. Tan, W.K. Loke, S. Wicaksono and S.F. Yoon \\ E. Ochoa and M. Gabás \\ T. Thomas and N. J. Ekins-Daukes \\ H. García, H. Castan and S. Dueñas \\ S. Estradé and F. Peiró
}

\begin{abstract}
Recent advances on the development of a $4 \mathrm{~J}$ latticematched dilute nitride solar cell for terrestrial and space applications are described. Modeling of the solar cell is carried out using a drift-diffusion model and material parameters extracted from ad hoc electro-optical characterization resulting in an efficiency prediction of $47 \%$ for concentrations of 1000 suns AM1.5d G173 spectrum and 33\% for 1x AM0. First experimental solar cell results of a dual-junction GaNAsSb/Ge solar cell and a triple-junction GaInP/Ga(In)As/GaNAsSb components of the full 4-Junction are shown.
\end{abstract}

Index Terms - dilute nitride materials, multijunction solar cells, characterization, modeling.

\section{INTRODUCTION}

Lattice-Matched (LM) multijunction solar cells including dilute nitride materials have the potential to reach very high efficiencies that may enable their use in both terrestrial and space applications. These kind of cells have already reached efficiencies of $44.0 \%$ at 942 suns by using a $\mathrm{GaInP} / \mathrm{GaAs} / \mathrm{GaInNAs}(\mathrm{Sb}) \cdot 3 \mathrm{~J}$ structure grown on inactive GaAs substrates [1]. For space applications, efficiencies of $31 \%$ (AM0) have been already announced [2]. These efficiencies can be exceeded by using a 4-junction device including a $\sim 1 \mathrm{eV}$ dilute nitride subcell grown on active germanium substrates. In order to undertake the development of a 4-Junction solar cell, we are investigating the combination of both MBE (to grow the dilute nitride) and MOVPE as a first step towards a full MOVPE process on 6-inch germanium wafers. This final approach would reduce the cost per wafer by using monolithically grown cells in a single process with enhanced efficiency and by augmenting the yield utilizing large Ge substrates. In this work, we show advances on the modeling and experimental developments towards a 4Junction including GaNAsSb as a first candidate for the $\sim \mathrm{leV}$ subcell.

\section{MODELING AND CHARACTERIZATION}

In order to develop a realistic model for the full $4 \mathrm{~J}$ including the dilute nitride subcell, we begin by validating our models by fitting typical experimental characterization curves (EQE, reflectivity, dark and light $\mathrm{I}-\mathrm{V}$ at various concentrations, among others) of conventional LM GaInP/Ga(In)As/Ge $3 \mathbf{J}$ solar cells with a multi-sun efficiency of $40 \%$. Then, we incorporate the GaNAsSb subcell using measured material parameters that serve as input data to the model [3]. The baseline structure corresponds to $\mathrm{GaInP} / \mathrm{Ga}$ (In)As/GaAsNSb/Ge with the following bandgaps $1.90,1.41,1.04,0.66 \mathrm{eV}$, respectively. The modeling was carried out within the frame of the TCAD simulator Atlas from Silvaco ${ }^{\circledR}[4]$.

A. Experimental performance and analysis of the $1 J$ GaNAsSb solar cell

Four GaNAsSb solar cell structures (see Fig. 1) with different absorber thicknesses and doping levels have been grown by Molecular Beam Epitaxy (MBE) with nominal compositions of $2.5 \%$ and $6.5 \%$ of nitrogen and antimony, respectively. Details on the growth can be found elsewhere [5]. Three structures have absorber (base) thicknesses of $1,1.5$ and 2 micrometers unintentionally doped (typically p-type with doping level of $5 \cdot 10^{15} \mathrm{~cm}^{-3}$ ) and the fourth sample 
corresponds to an absorber thickness of 1.5 micrometers with a doping level $(\mathrm{Be})$ of $5 \cdot 10^{17} \mathrm{~cm}^{-3}$. The emitter is an n-type (Si) region with a doping level of $1 \cdot 10^{17} \mathrm{~cm}^{-3}$ and $100 \mathrm{~nm}$ thick. The p-n junction is surrounded by highly doped GaAs front and back surface field layers as can be seen in Fig. 1. The epiwafers were manufactured into $0.1 \mathrm{~cm}^{2}$ active area solar cells and characterized by means of quantum efficiency, reflectivity and current-voltage measurements [3].

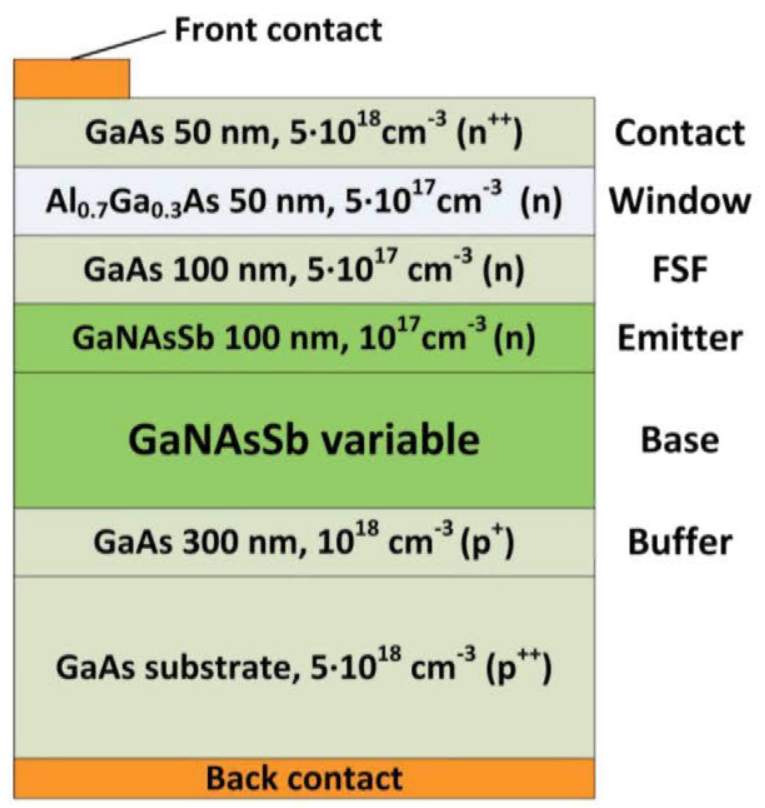

Fig. 1. Schematic structure of GaNAsSb single junction solar cell.

The performance of the GaNAsSb solar cells without antireflection coating (ARC) has been evaluated experimentally and theoretically. On the one hand, we have found that the best performance in terms of quantum efficiency (see Fig. 2) corresponds to a $1 \mu \mathrm{m}$ absorber thickness solar cell. This subcell would produce a photocurrent (calculated from the convolution of $\mathrm{EQE}$ and the spectral irradiance in the range of $880-1200 \mathrm{~nm})$ of 6.25 and $7.75 \mathrm{~mA} / \mathrm{cm}^{2}$ for terrestrial and space spectrums, respectively. This is about $60-65 \%$ of the photocurrent required to be useful in a 4-Junction solar cell for any spectrum. The low internal quantum efficiency (IQE) values in the useful range $(880-1200 \mathrm{~nm})$ are mainly due to the low diffusion length of carriers measured for these materials as a consequence of nitrogen-related defects [5]. As a consequence, solar cells with very low doping levels at the absorber are typically used, since their photocurrent is dominated by electric field-assisted collection [3,7].

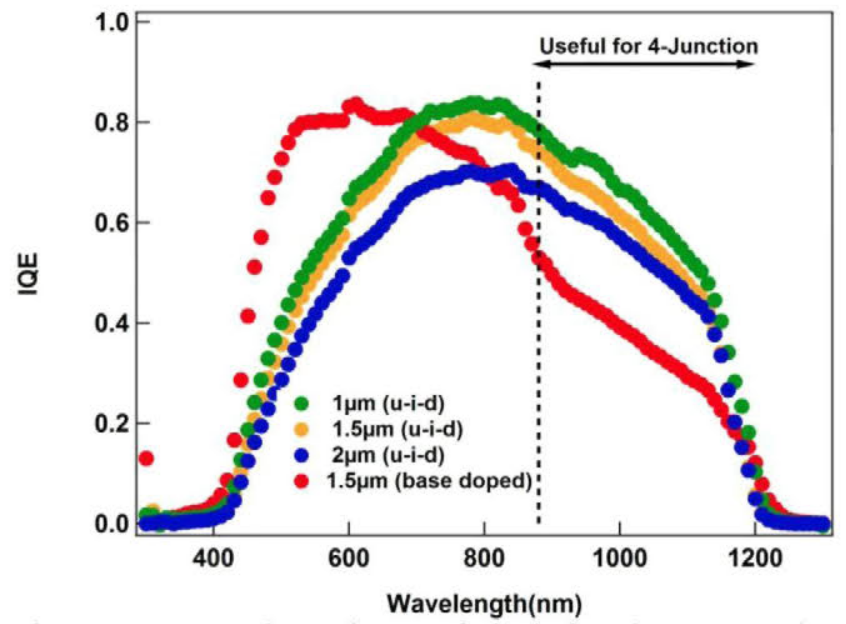

Fig. 2. (a) Experimental IQE of single junction GaNAsSb solar cells with different absorber thickness. Red circles correspond to a doped absorber $\left(5 \cdot 10^{17} \mathrm{~cm}^{-3}\right)$ while the rest correspond to unintentionally doped absorbers.

On the other hand, it has been found that the IQE drops as thickness increases as can be seen in Fig. 2. This IQE degradation with thickness cannot be explained by electricfield assisted collection and low diffusion lengths. Nonuniformity in material composition or doping levels in the growth direction may be responsible for this behaviour. Accordingly, this issue is still under investigation. Interestingly, the doped-absorber solar cell has the worst performance between $880-1200 \mathrm{~nm}$ while its response improves between $400-700 \mathrm{~nm}$. This result is qualitatively in agreement with the fact that the photocurrent is electric fieldassisted. A better response at the emitter region is caused by a strong electric field (a minimum value of $40 \mathrm{kV} / \mathrm{cm}$ has been calculated from simulations) and a fully depleted emitter due to the lower doping level $\left(1 \cdot 10^{17} \mathrm{~cm}^{-3}\right)$ with respect to the base $\left(5 \cdot 10^{17} \mathrm{~cm}^{-3}\right)$. The depletion width at the base reduces from 500 to $100 \mathrm{~nm}$ being the main cause of the poor response at higher wavelengths (880-1200nm).

Using the modeling, we can break down the spectral response contribution from each part of the solar cell. Details on the modeling validation can be found in [3]. As can be seen in Fig. 3, most of the contribution in the $1 \mu \mathrm{m}$ absorber solar cell comes from the space charge region (orange line) corresponding to a $70 \%$ of the total spectral response (black line) within the useful wavelength range $(880-1200 \mathrm{~nm})$ for a 4-Junction device. In the case of the quasi-neutral regions, diffused carriers contribute to the total photocurrent with about $20 \%$ and $10 \%$ for the base and emitter, respectively. This high contribution from the space charge region is in agreement with electric-field assisted collection and limits the performance not only in terms of photocurrent, but also in fill factor [3]. 


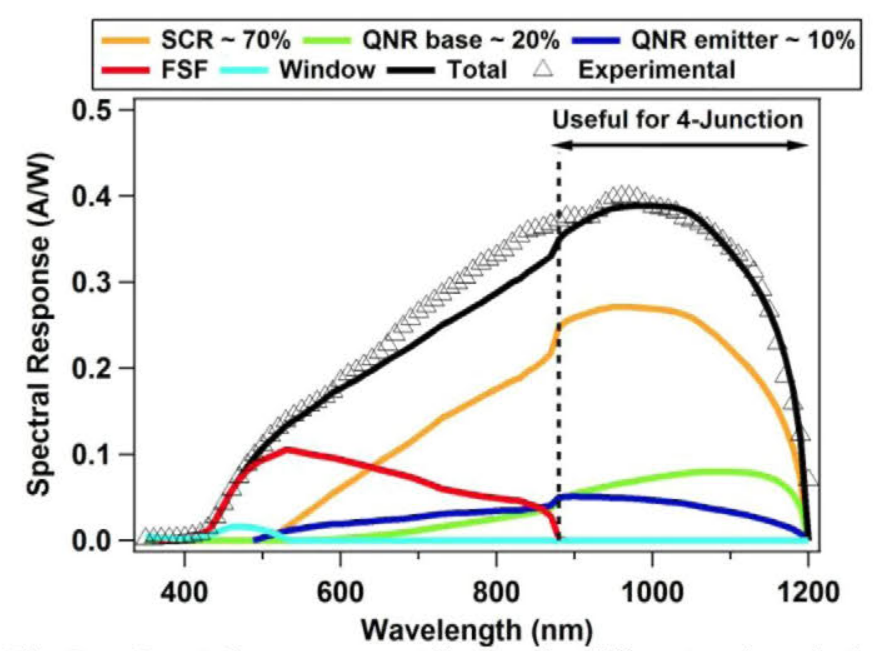

Fig. 3. Spectral response contribution for different regions in the simulated GaNAsSb solar cell ( $1 \mu \mathrm{m}$ absorber thickness). FSF stands for front surface field layers, respectively. The rest of the layers (contact, buffer and substrate) are not shown because their contribution to the total photocurrent is negligible. The inset shows the structure grown and simulated. The depletion width in the base region for this structure is about $600 \mathrm{~nm}$ at short circuit conditions. SCR stands for space charge region while QNR for quasi-neutral region.

In the case of illumination current-voltage characteristics, Table I summarizes the solar cell parameters measured under 1-sun AM1.5d spectrum. As can be seen, the highest fill factor measured is about $65 \%$ being lower than expected $(>70 \%)$ mainly due to the electric-field collection dependence. Open circuit voltages up to $420-430 \mathrm{mV}$ have also been measured. Considering a bandgap of $1.04 \mathrm{eV}$, as calculated from $\mathrm{EQE}$ curves, the bandgap-voltage offset $\left(\mathrm{E}_{\mathrm{G}}-\mathrm{qV} \mathrm{V}_{\mathrm{OC}}\right)$ is around $600 \mathrm{mV}$ indicating that the material quality has to be improved. For solar cells with very high material quality, $400 \mathrm{mV}$ of bandgap-voltage offset is expected [8].

TABLE I

Solar cell parameters of the GaNAsSb single junction solar cells. *The solar cell $\mathrm{N} 2980$ has a base doping $\mathrm{N}_{\mathrm{B}}=5 \cdot 10^{17} \mathrm{~cm}^{-3}$.

\begin{tabular}{ccccc} 
Sample & $\begin{array}{c}\text { Base } \\
\text { thickness } \\
(\mu \mathrm{m})\end{array}$ & $\begin{array}{c}\mathrm{Jsc} \\
(\mathrm{mA} / \mathrm{cm} 2)\end{array}$ & Voc $(\mathrm{V})$ & $\mathrm{FF}$ \\
\hline $\mathrm{N} 2976$ & 2 & 15.1 & 0.43 & $64.3 \%$ \\
$\mathrm{~N} 2978$ & 1.5 & 14.3 & 0.42 & $48.5 \%$ \\
$\mathrm{~N} 2980$ & $1.5^{*}$ & 13.3 & 0.37 & $65.2 \%$ \\
$\mathrm{~N} 2982$ & 1 & 16.2 & 0.42 & $56.6 \%$
\end{tabular}

B. Projections of 4-Junction performance including a GaNAsSb subcell for space and concentrator applications

As explained in the previous section, the experimental GaNAsSb subcell developed exhibits a $V_{O C}$ that would be useful to increase the efficiency of standard GaInP/GaAs/Ge triple-junction solar cells if similar $\mathrm{J}_{\mathrm{SC}}$ and $\mathrm{FF}$ were achieved. However, the photocurrent in the GaNAsSb subcell is currently too low to be useful in such 4-junction solar cell. One of the pathways to improve the GaNAsSb performance is to increase the diffusion length of minority carriers $\left(\mathrm{L}_{\mathrm{D}}\right)$. As an example, Fig. 4 shows that a ten times increase in $L_{D}$ (pink line) from the present material $L_{D}[3]$ yields a significant enhancement on the GaNAsSb EQE ( $85 \%$ average). The photocurrent is boosted about 3.8 and $4 \mathrm{~mA} / \mathrm{cm}^{2}$ for terrestrial and space spectrum, respectively while the Voc increases $\sim 100 \mathrm{mV}$ and the FF goes up to $72 \%$. These solar cell parameters are comparable to the ones already achieved experimentally in other dilute nitrides by means of thermal annealing treatments [9] and/or specific growth conditions related to the beam equivalent pressure ratios of $\mathrm{V} / \mathrm{III}$ elements [10]. Accordingly, in the following simulations we will use a $\mathrm{L}_{\mathrm{D}}$ augmented by a factor of 10 in the GaNAsSb subcell in order to estimate the potential and evaluate the performance of the $4 \mathrm{~J}$ solar cell.

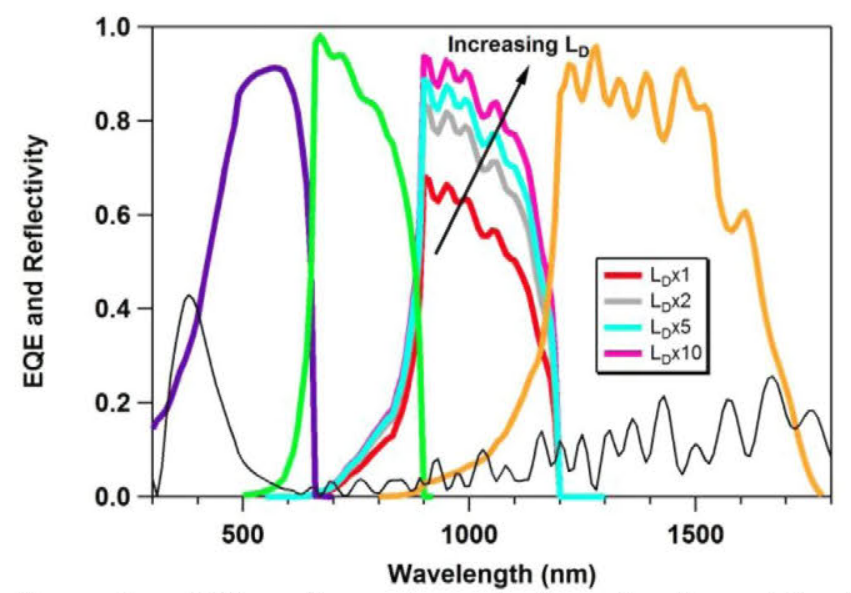

Fig. 4. EQE of each subcell of the 4-Junction GaInP/GaInAs/GaNAsSb/Ge. The EQE's of the GaNAsSb subcell is depicted for four different factors of the current diffusion length ( 1 , 2,5 and 10). The lower response in the GaAs subcell is due to its thinner thickness $(1.25 \mu \mathrm{m})$ than usual. A bilayer ARC of $\mathrm{MgF}_{2}(110 \mathrm{~nm}) / \mathrm{ZnS}(70 \mathrm{~nm})$ has been assumed and the corresponding reflectivity of the 4-Junction is shown as a black solid line.

Table II shows the photocurrent availability for a total light collection (ideal) in each subcell for both terrestrial and space spectrums. The photocurrents produced for each subcell calculated from their IQE curves are also shown in order to evaluate their collection efficiency. By looking at the collection efficiency values (percentage values of the available photocurrent between parenthesis in Table II), the GaAs subcell is the one that exhibits the higher performance with about $95 \%$ of the photons collected for any spectrum. The $\mathrm{GaInP}$ and GaNAsSb subcells utilize photons with similar efficiency being greater for the terrestrial spectrum than for the space one $(\sim \Delta 3 \%)$. In the case of Ge subcell, it is the one showing the lowest collection efficiency, $75.3 \%$ and $81 \%$ for terrestrial and space spectrum, respectively. Accordingly, there is still room for improvement in any subcell, especially in Ge and to a lower extent in GaInP and GaNAsSb ones. 
TABLE II

Available photocurrents for each subcell of the $4 \mathrm{~J}$ structure for space and terrestrial spectrum. The calculated photocurrents from simulated IQE curves (see Fig. 5) are also shown for both spectrums. The values in parenthesis correspond to the collection efficiency of each subcell assuming no reflection losses.

\begin{tabular}{ccccccc}
\hline \multirow{2}{*}{ Subcell } & \multicolumn{2}{c}{$J_{\mathrm{sc}}(\mathrm{AM} 1.5 \mathrm{~d})\left[\mathrm{mA} / \mathrm{cm}^{2}\right]$} & \multicolumn{3}{c}{$J_{\mathrm{sc}}(\mathrm{AMO})\left[\mathrm{mA} / \mathrm{cm}^{2}\right]$} \\
& Available & From IQE & Available & \multicolumn{2}{c}{ From IQE } \\
\hline \hline $\begin{array}{c}\text { GalnP } \\
{[1.90 \mathrm{eV}]}\end{array}$ & 16.1 & 14.0 & $(86.8 \%)$ & 21.9 & 18.3 & $(83.4 \%)$ \\
$\begin{array}{c}\text { GalnAs } \\
{[1.41 \mathrm{eV}]}\end{array}$ & 16.9 & 16.3 & $(96.4 \%)$ & 18.4 & 17.6 & $(95.6 \%)$ \\
$\begin{array}{c}\text { GaNAsSb } \\
{[1.04 \mathrm{eV}]} \\
\mathrm{Ge}\end{array}$ & 13.6 & 11.7 & $(86.0 \%)$ & 17.7 & 14.7 & $(83.3 \%)$ \\
$\begin{array}{c}\mathrm{G} .66 \mathrm{eV}] \\
\text { Average }\end{array}$ & 15.5 & 11.6 & $(75.3 \%)$ & 20.9 & 16.9 & $(81.0 \%)$ \\
\hline
\end{tabular}

In order to significantly exceed the efficiency of a $3 \mathrm{~J}$ by using a $4 \mathrm{~J}$ solar cell, the additional junction must be close to the state-of-the-art photocurrent values of $3 \mathrm{~J}$ solar cells. Typically, a $\mathrm{J}_{\mathrm{SC}}$ of 14 and $17 \mathrm{~mA} / \mathrm{cm}^{2}$ is desirable for terrestrial and space spectra, respectively. As can be seen in Table II, the average value which corresponds to the limiting photocurrent (once the photocurrent is properly distributed) is lower than the desired $\mathrm{J}_{\mathrm{SC}}$ in any spectrum. In order to improve this photocurrent, the spectral response of the device is examined in Fig. 5 where carrier collection, recombination and reflection losses are shown together with the corresponding subcell EQEs and the reflectivity of the 4Junction solar cell.

There are three important spectral regions where losses are especially significant where the IQE drops below $85 \%$ as illustrated in Fig. 5: 1) $\lambda<500 \mathrm{~nm}$, 2) $1100 \mathrm{~nm}<\lambda<1200 \mathrm{~nm}$ and 3) $1500<\lambda>1800 \mathrm{~nm}$. In region 1 , the high $\mathrm{J}_{\mathrm{SC}}$ loss $(\sim 15 \%)$ is mainly due to surface recombination and window/emitter interface recombination. In region 2 , the loss is mainly related to the diffusion length of carriers in GaNAsSb while region 3 is related to the indirect bandgap of $\mathrm{Ge}$ and its lower absorption coefficient and insufficient diffusion lengths. In the case of $\mathrm{Ge}$, the use of advanced $\mathrm{Ge}$ with that uses low-doped substrates with larger diffusion lengths has been already reported [11]. This may be one of the pathways to improve the long wavelengths response in Ge to maximize the efficiency output in space while for concentrator applications the series resistance may become an issue due to the low-doped Ge substrate.

Assuming no further improvements in the present device, it is important to mention that there is a pathway to mitigate the recombination losses in terms of photocurrent. This is of special importance in the case of $3^{\text {rd }}$ and $4^{\text {th }}$ junctions as shown previously. For example, the amount of photons lost by recombination at specific wavelength region (close to the bandgap edges) can be mitigated by making other subcell with higher collection efficiency at that wavelength range to collect those photons. For example, in region $2(1100 \mathrm{~nm}<\lambda<$ $1200 \mathrm{~nm}$ ), the GaNAsSb subcell has high recombination losses as can be deduced from the large blue region and the decreasing $\mathrm{EQE}$. However, germanium is capable to take advantage because it is more efficient than GaNAsSb in this wavelength range which has been deduced from the $90 \%$ average IQE of Ge (not shown). The same reasoning applies to favor the GaNAsSb, but in the GaAs region close to its bandgap. In this region, the IQE of GaNAsSb is higher than any other higher wavelength. Obviously, this would involve the thinning of the corresponding junctions but this may also be beneficial to get higher $V_{O C}$ 's .

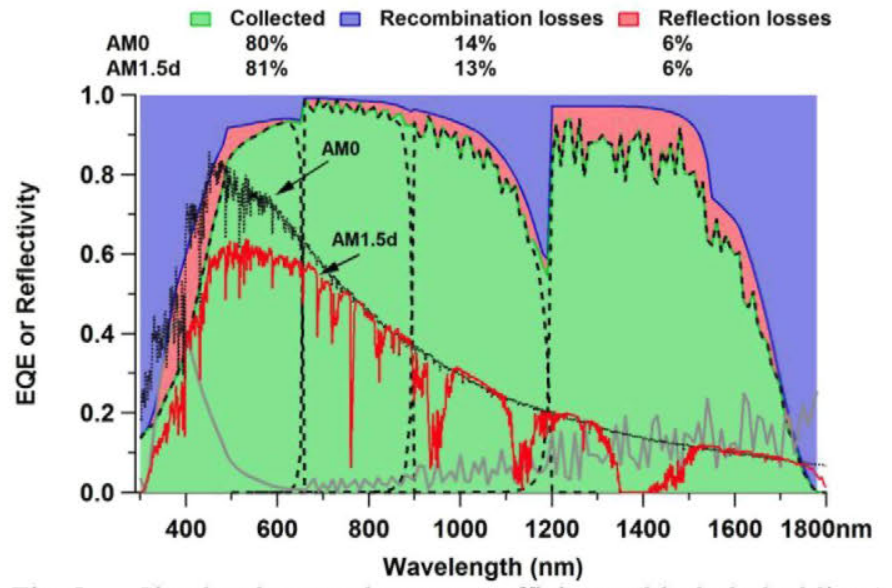

Fig. 5. Simulated external quantum efficiency (black dashed lines) of the 4-Junction subcells. The corresponding reflectivity is shown by a solid gray line. The region where photons are lost by recombination is shown in blue while the region corresponding to reflection loss is shown in red. Each junction has enough thickness to absorb above $98 \%$ average of the incoming light in their corresponding wavelength range. In the legend, a summary of collected and lost photocurrent is depicted.

Finally, we have also computed light I-V curves as a function of subcell thicknesses in order to optimize the performance of the solar cell. In the case of concentrator applications, we found a peak efficiency of $44 \%$ at 1000 suns for a representative device with a shadowing factor of $3 \%$ and $\mathrm{Jsc}=12.8 \mathrm{~mA} / \mathrm{cm}^{2}, \mathrm{Voc}=4 \mathrm{~V}$ and $\mathrm{FF}=86 \%$ [3]. The present ARC has not been optimized (as shown previously) in the whole spectrum range. In this respect, further improvement can be achieved by taking into account the low reflectivity offered by a broadband ARC (i.e. $\mathrm{R}<5 \%$ in the whole spectrum range) [12]. By including this improved ARC and tweaking the structure, a peak efficiency $\sim 47 \%$ at 1000 suns (mainly boosted by $\mathrm{Jsc}=13.7 \mathrm{~mA} / \mathrm{cm}^{2}$ ) can be achieved [3]

Regarding space applications, we have set the top cell as the limiting photocurrent which is also a good rule for EOL since GaAs solar cells are the most affected by the radiation environment while GaNAsSb subcell has not been tested yet. The estimated efficiency for this structure is $31.4 \%$ with a $\mathrm{Jsc}=16.5 \mathrm{~mA} / \mathrm{cm}^{2}$, Voc $=3.2 \mathrm{~V}$ and $\mathrm{FF}=83 \%$. By using the improved ARC and adjusting the structure, there is potential to boost the efficiency above $33 \%$. Despite the numbers given 
here, it is important to note that the final values are very dependent on the spectral response of each subcell and the ARC design. There is some degree of flexibility if one can vary the top cell and dilute nitride bandgaps as well as the thickness of each junction. Finally, the ARC must be designed taking into account the spectral response of each subcell.

\section{PRELIMINARY SOLAR CELL MANUFACTURING TOWARDS 4- JUNCTION SOLAR CELL}

\section{A. GaNAsSb/Ge dual-junction solar cell}

In the quest for the $4 \mathrm{~J}$ solar cell, independent optimization of some processes and structures is required. Currently, we have already grown the $3^{\text {rd }}$ and $4^{\text {th }}$ subcells by MBE and MOVPE, respectively (see Fig. 6).

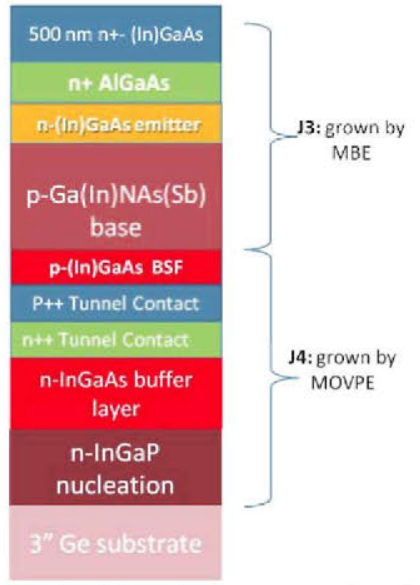

Fig. 6. GaNAsSb /Ge dual junction structure developed for optimization purposes.

Fig. 7 shows the IQE response of this dual junction solar cell $\mathrm{GaNAsSb} / \mathrm{Ge}$ (red) together with the single junction solar cells grown for benchmarking (black). As can be seen, the Ge subcell grown by MOVPE has equivalent performance to the single junction Ge reference solar cell. This indicates that the state-of-the-art performance of the Ge subcell remains after the MBE growth of the dilute nitride solar cell. In the case of the GaNAsSb solar cell, some degradation has been observed as well as a blue-shifting in the cut-off wavelength of the IQE (about $35 \mathrm{meV}$ difference). The reasons are currently under study, but a significant lattice-mismatch of the dilute nitride subcells to the Ge substrate, which causes relaxation in this subcell and formation of defects, is pointed out as the most probable cause. In order to achieve lattice-matching of GaNAsSb to Ge, some slight changes in the composition of $\mathrm{N}$ and $\mathrm{Sb}$ are required. XRD calibration and theoretical calculations have been performed to achieve lattice matching of GaNAsSb to Ge. Accordingly, SIMS and RBS measurements are currently underway to determine the exact composition of the dilute nitride material and also to confirm that is the main cause behind the different performance between GaNAsSb on Ge and the single junction reference solar cells.

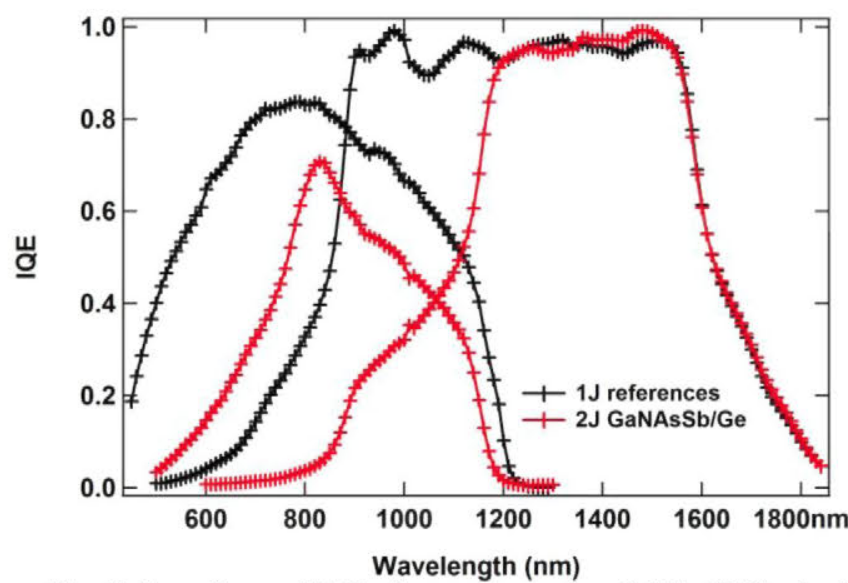

Fig. 7. Experimental IQE of a representative GaNAsSb/Ge dual junction solar cell (red) and their corresponding single junction solar cells grown for benchmarking (black).

Interestingly, the subsequent growth of GaNAsSb on $\mathrm{Ge}$ templates does not exhibit significant degradation of the open circuit voltage. $\mathrm{V}_{\mathrm{OC}}$ 's dual junction in the range of 600 $650 \mathrm{mV}$ have been measured corresponding roughly to the sum of each subcell $\mathrm{V}_{\mathrm{OC}}$. About $400-450 \mathrm{mV}$ is expected to be produced by GaNAsSb while the Ge subcell is expected to provide $240 \mathrm{mV}$.

Presently, we are also optimizing the annealing process of the dilute nitride in order to improve its minority carrier properties while preserving the good properties of the $\mathrm{Ge}$ subcell as the ones shown in Fig. 7. This optimization takes into consideration the thermal load of the subsequent MOVPE process for the growth of the first and second subcells.

Finally, the proof of concept of the 4-Junction solar cell ( $\mathrm{GaInP} / \mathrm{Ga}(\mathrm{In}) \mathrm{As} / \mathrm{GaNAsSb} / \mathrm{Ge}$ ) has been already achieved by combining MOVPE+MBE technologies. The performance of this solar cell is currently under analysis.

\section{SUMMARY AND CONCLUSIONS}

The potential of 4-junction lattice-matched dilute nitride solar cells for both terrestrial and space applications has been studied. A realistic modeling has been developed departing from an extensive dilute nitride material characterization. By using minority carrier properties that achieve similar performance of dilute nitride solar cells found in literature, the optimization results in efficiencies above $47 \%$ at 1000 suns and $33 \%$ for concentration and space applications, respectively. In order to realize these efficiencies, further improvement of our present dilute nitride material quality grown is required and corresponding annealing treatments are currently under development. Finally, preliminary $2 \mathrm{~J}$ $\mathrm{GaNAsSb} / \mathrm{Ge}$ solar cells have been fabricated by combining MOVPE $+\mathrm{MBE}$ technologies. It has been shown that the integration of subcell components using this multi-reactor approach produces no degradation of the Ge subcell. The achievement of the proof of concept of the 4-Junction solar 
cell on Ge has been already achieved and the evaluation of its performance is ongoing. This is encouraging towards the attainment of a fully MOCVD process for the growth of the 4junction structure.

\section{ACKNOWLEDGEMENT}

This work has been supported by the European Commission by means of the LONGESST project (FP7 grant Agreement Number 607153), by the Spanish MINECO through the projects TEC2014-54260-C3-3-P and TEC2014-54260-C3-1P, PCIN-2015-181-C02-01, PCIN-2015-181-C02-02 by the Comunidad de Madrid through the project MADRID-PV (S2013/MAE-2780) and by the project MAT2013-41506. IQE has subcontracted NTU within the frame of the LONGESST project.

\section{REFERENCES}

[1] http://www.semiconductortoday.com/news items/2012/OCT/SOLARJUNCTION 151012. $\underline{\mathrm{html}}$

[2] http://www.sj-solar.com/about us/space.php

[3] M. Ochoa, et al., "Modelling of Lattice Matched Dilute Nitride 4-junction Concentrator Solar Cells on Ge Substrates", submitted to AIP Conference Proceedings, 2016.

[4] Silvaco software, "Atlas User's Manual", 2015.

[5] K. H. Tan, S. Wicaksono, W. K. Loke, D. Li, S. F. Yoon, E. A. Fitzgerald, S. A. Ringel, and J. S. Harris Jr., "Molecular beam epitaxy grown GaNAsSb $1 \mathrm{eV}$ photovoltaic cell," J. Cryst. Growth, vol. 335, no. 1, pp. 66-69, Nov. 2011.

[6] T. Thomas, M. Fuhrer, D. A. Alvarez, N. Ekins-Daukes, K. H. Tan, S. Wicaksono, W. K. Loke, S. F. Yoon, and A. Johnson, "GaNAsSb 1-eV solar cells for use in lattice-matched multijunction architectures," in Conference record of the 40th IEEE Photovoltaic Specialists Conference, 2014, pp. 0550-0553.

[7] D. J. Friedman, A. J. Ptak, S. R. Kurtz, and J. F. Geisz, "Analysis of depletion-region collection in GaInNAs solar cells," Conf. Rec. Thirty-first IEEE Photovolt. Spec. Conf. 2005., no. February, pp. 4-7, 2005.

[8] R. R. King, A. Boca, W. Hong, X. Q. Liu, D. Bhusari, D. Larrabee, K. M. Edmondson, D. C. Law, C. M. Fetzer, S. Mesropian, and N. H. Karam, "Band-Gap-Engineered Architectures for High-Efficiency Multijunction Concentrator Solar Cells Acknowledgments," 24th Eur. Photovolt. Sol. Energy Conf. 21-25 Sept. 2009, Hamburg, pp. 55 - 61, 2009.

[9] N. Miyashita, N. Ahsan, and Y. Okada, "Generation and collection of photocarriers in dilute nitride GaInNAsSb solar cells," Progress in Photovoltaics Research and Applications, vol. 24, no. 1, pp. 28-37, Jun. 2016.

[10] V. Polojärvi, A. Aho, A. Tukiainen, M. Raappana, T. Aho, A. Schramm, and M. Guina, "Influence of As/group-III flux ratio on defects formation and photovoltaic performance of GaInNAs solar cells," Sol. Energy Mater. Sol. Cells, vol. 149, pp. 213220, 2016.

[11] R. Hoheisel, J. Fernandez, F. Dimroth, and A. W. Bett, "Investigation of radiation hardness of germanium photovoltaic cells," IEEE Trans. Electron Devices, vol. 57, no. 9, pp. 2190$2194,2010$.

[12] E. E. Perl, C. T. Lin, W. E. McMahon, D. J. Friedman, and J. E. Bowers, "Ultrabroadband and wide-angle hybrid antireflection coatings with nanostructures," IEEE J. Photovoltaics, vol. 4, no. 3, pp. 962-967, 2014. 